\title{
A new family of bridge functions for electrolyte solutions
}

\author{
F.O.Raineri, G.Stell \\ Department of Chemistry, State University of New York at Stony Brook, \\ Stony Brook, New York 11794-3400, USA
}

Received November 5, 2001

\begin{abstract}
We present a new set of closures for restricted models of electrolyte solutions at the McMillan-Mayer level that improve upon the Hypernetted Chain prediction for the ion-ion pair correlation functions. The improvement is accomplished by proposing simple functional forms for the bridge functions and the specification of certain adjusting parameters according to several criteria. Under the new closures, and unlike the HNC case, the "sum" direct correlation function, which is crucial for determining the stability of the solution with respect to phase separation, remains finite at thermodynamic states along the spinodal and at the critical point.
\end{abstract}

Key words: electrolyte solutions, restricted model, structure, thermodynamics, bridge functions, thermodynamic consistency

PACS: $61.20 . \mathrm{Gy}, 61.20 . \mathrm{Qg}, 64.60 . \mathrm{Fr}, 64.70 . \mathrm{Ja}, 64.75 .+\mathrm{g}$

\section{Introduction}

The accurate prediction of the structure and thermodynamic properties of electrolyte solutions over a wide range of thermodynamic states has been the focus of an enormous number of studies. In spite of these efforts, there are still a number of issues that require more development, especially those concerning the description of the structure and thermodynamic properties of an electrolyte solution close to criticality (i. e., the critical point associated with the unmixing of an ionic solution into two liquid phases of different electrolyte concentration). Another relevant issue is the need of an improved description of the structure and thermodynamic properties for aqueous solutions of 2-2 electrolytes (and also for electrolytes of higher charge) at low concentrations.

The vast majority of the efforts have focussed on the calculation of the structure and other properties of electrolyte solutions by statistical mechanical methods at the McMillan-Mayer level [1]: integral equations and computer simulation. In the first approach the ion-ion pair correlation functions $g_{j l}(r)$ are calculated by solving an appropriate set of Ornstein-Zernike (OZ) equations, that is supplemented with 
a set of additional relations between the indirect $\left\{h_{j l}(r)\right\}$ and the direct $\left\{c_{j l}(r)\right\}$ correlation functions $[2,3]$. Because of their role in setting the mathematical problem determinate, the additional relations are referred to as closure relations or integral equation closures.

Among the closure relations proposed for ionic systems, the hypernetted chain (HNC) closure [2,3] has probably been the closure most thoroughly investigated. The HNC closure predicts the structural and thermodynamic properties of 1-1 electrolytes quite accurately up to concentrations greater that $1 \mathrm{~mol} \mathrm{dm}^{-3}$. For $2-2$ electrolytes, however, this closure predicts a spurious peak in the like-sign (i. e., cationcation or anion-anion) correlation functions, which is absent in the correlation functions extracted from computer simulation calculations [4]. At the same time, results obtained from molecular dynamics computer simulations indicate that the height $\left(g_{12}^{\max }\right)_{\mathrm{MD}}$ of the peak in the cation-anion correlation function is considerably larger than the corresponding quantity $\left(g_{12}^{\max }\right)_{\mathrm{HNC}}$ obtained with the HNC closure $[5,6]$. A somewhat less known problem is that the direct correlation functions $c_{j l}(r)$ under the HNC closure become long-ranged as the state of the solution approaches the critical point (or, more generally, the stability limit of the solution with respect to phase separation) [7].

Recently we proposed an integral equation closure for general models of binary electrolytes that gives direct correlation functions $c_{j l}(r)$ that remain of finite range at every thermodynamic state [7]. The new closure is formulated by decomposing the density fluctuation of each ionic species into contributions to two mutually orthogonal types of fluctuations: the charge density fluctuations and the neutral density fluctuations. The source in the ion-ion direct correlation functions responsible for the long-range behaviour close to the critical point is eliminated in the closure by appropriately choosing the bridge function. The particular form of the bridge function, which is reminiscent of the Percus-Yevick (PY) type bridge function [see equation (18) below], explains the name, HNC/PY, assigned to the closure. A shortcoming of the proposed HNC/PY closure is that it systematically gives $\left(g_{12}^{\max }\right)_{\mathrm{HNC} / \mathrm{PY}}$ smaller than $\left(g_{12}^{\max }\right)_{\mathrm{HNC}}$ and $\left(g_{12}^{\max }\right)_{\mathrm{MD}}$.

The purpose of this paper is to propose and investigate a new family of integral equation closures for electrolyte solutions that are constructed using the HNC/PY closure as the starting building block. The closures are obtained by simply extending the bridge function associated with the HNC/PY closure with supplementary terms of carefully selected form. In particular, the functional forms of the additional bridge function terms are extracted from the asymptotic behaviour of the direct correlation functions under the HNC/PY closure. Clearly this strategy guarantees the property (shared with the HNC/PY closure) that the new closures remain of finite range close to the critical point.

Although the HNC/PY closure was originally formulated for general solution models of binary electrolytes at the McMillan-Mayer level [7], here we confine the presentation to the simpler case of solutions of restricted or symmetric binary electrolyte models (see the next section for details). Furthermore, with respect to the performance of the new closures, in this work we consider only temperature and 
concentration states where the electrolyte solution is stable.

The outline of the rest of the paper is as follows. In section 2 we discuss some theoretical features of the HNC and the HNC/PY integral equation closures. Unlike in the original presentation [7], here we use the language of the sum and difference ion-ion correlation functions that is so convenient for problems involving symmetric binary electrolytes. In this section we also introduce the new integral equation closures $\mathrm{HNC} / \lambda_{1}$ and $b_{D} / \lambda_{1}$ by adding new terms to the bridge functions in the $\mathrm{HNC} / \mathrm{PY}$ closure. In section 3 we report calculations that test the performance of the $\mathrm{HNC} / \lambda_{1}$ closure for models of aqueous solutions of a $2-2$ electrolyte at $298.15 \mathrm{~K}$. The results are compared with the results obtained from calculations with the HNC and HNC/PY closures, as well as with the results obtained from molecular dynamics computer simulations of the same solution models by Smith et al. [5]. Finally, in section 4 we briefly discuss the results obtained under the $\mathrm{HNC} / \lambda_{1}$ closure, and suggest alternatives for further improvement (the $b_{D} / \lambda_{1}$ closure and another extension, the $\mathrm{HNC} / \lambda_{2}$ closure). We also compare our approach with previous work on the development of bridge functions appropriate for electrolyte solutions.

\section{Theory}

Our starting point is the well known McMillan-Mayer level expression $[2,3]$

$$
h_{j l}(r)=\exp \left(-\beta \bar{u}_{j l}(r)+t_{j l}(r)+b_{j l}(r)\right)-1, \quad j, l=1,2
$$

for the ion-ion correlation functions, where the indices $j$ and $l$ refer to the ionic species: 1 for the cations and 2 for the anions. As usual, $\beta=\left(k_{\mathrm{B}} T\right)^{-1}$ has the meaning of the inverse of the temperature expressed in energy units $\left(k_{\mathrm{B}}\right.$ is the Boltzmann constant).

We recall that the indirect correlation function $h_{j l}(r)$ is related to the ion-ion radial distribution function $g_{j l}(r)$ [which is proportional to the probability that two ions, one of species $j$ and the other of species $l$, are separated by a distance $r$ in the ionic solution] according to the equation $h_{j l}(r)=g_{j l}(r)-1$. The other functions in equation (1) are $\bar{u}_{j l}(r)$, the solvent-averaged potential between two ions of species $j$ and $l$ separated by a distance $r$ at infinite dilution $[1,2] ; t_{j l}(r) \equiv h_{j l}(r)-c_{j l}(r)$, in which $c_{j l}(r)$ is the ion-ion direct correlation function; and finally the ion-ion bridge function $b_{j l}(r)[2,3]$. The sets of functions $\left\{h_{j l}(r)\right\}$ and $\left\{c_{j l}(r)\right\}$ are further interrelated through (see below) the Ornstein-Zernike equations $[2,3]$.

As mentioned in the introduction, in this study we focus on the case of a symmetric (or restricted) model of an electrolyte solution. In such a model the electrolyte dissociates into equal numbers of cations and anions. Furthermore, the cation and anion species are entirely equivalent, except for their charges $z_{1} e$ and $z_{2} e$, which are of the same magnitude but of opposite signs $\left(z_{1}=-z_{2} ; e\right.$ is the charge of a proton). Quite generally, then, we write the McMillan-Mayer level ion-ion solvent averaged potentials $\bar{u}_{j l}(r)$ as the sum of a short-range contribution $\bar{u}^{*}(r)$, that is the same for every $j l$ ionic pair-type, and a long-range Coulombic contribution whose sign 
depends on whether the labels $j$ and $l$ refer to the same or to different ionic species:

$$
\bar{u}_{j l}(r)=\bar{u}^{*}(r)+(-)^{j+l} \frac{z^{2} e^{2}}{\epsilon_{0} r}, \quad j, l=1,2 .
$$

It should be noted that in the McMillan-Mayer level description, the Coulomb interaction between the charges is attenuated by the dielectric constant $\epsilon_{0}$ of the pure solvent. A particular but important case of equation (2) is the restricted primitive model, for which $\bar{u}^{*}(r)$ is a hard sphere potential. In this study, however, the discussion will not be limited to that model, but instead it will consider more general forms of $\bar{u}^{*}(r)$.

For the restricted electrolyte models represented by equation (2) it is advantageous to express any ion-ion function $F_{j l}$ [such as any of the functions that occur in equation (1)] in terms of the "sum" (subscript $S$ ) and difference (subscript $D$ ) functions:

$$
F_{S}(r) \equiv\left[F_{11}(r)+F_{12}(r)\right] / 2, \quad F_{D}(r) \equiv\left[F_{11}(r)-F_{12}(r)\right] / 2 .
$$

With this notation, equation (1) for $j l=11,12,21$, and 22 may be cast in the more succinct form

$$
\begin{aligned}
& h_{S}(r)=\mathrm{e}^{-\beta \bar{u}^{*}(r)+t_{S}(r)+b_{S}(r)} \cosh \mathcal{D}(r)-1, \\
& h_{D}(r)=\mathrm{e}^{-\beta \bar{u}^{*}(r)+t_{S}(r)+b_{S}(r)} \sinh \mathcal{D}(r),
\end{aligned}
$$

where we have introduced the auxiliary function

$$
\mathcal{D}(r) \equiv-\beta \bar{u}_{D}(r)+t_{D}(r)+b_{D}(r)
$$

Notice that because $\bar{u}^{*}(r)$ is the same function for every $j l$ pair, we have used in writing equations (4) and (5) that $\bar{u}_{S}(r)=\bar{u}^{*}(r)$, the short-range part of the ion-ion potential energy of interaction. Correspondingly, we note that the nature of $\bar{u}_{D}(r)$ is purely Coulombic: $\bar{u}_{D}(r)=z^{2} e^{2} / \epsilon_{0} r$.

Equations (4) and (5) may also be rearranged to display the sum and the difference direct correlation functions explicitly:

$$
\begin{aligned}
& c_{S}(r)=\mathrm{e}^{-\beta \bar{u}^{*}(r)+t_{S}(r)+b_{S}(r)} \cosh \mathcal{D}(r)-1-t_{S}(r), \\
& c_{D}(r)=\mathrm{e}^{-\beta \bar{u}^{*}(r)+t_{S}(r)+b_{S}(r)} \sinh \mathcal{D}(r)-t_{D}(r) .
\end{aligned}
$$

In terms of the Fourier transforms of the sum and difference functions, the Ornstein-Zernike equations at the McMillan-Mayer level are

$$
\begin{aligned}
& \widetilde{h}_{S}(k)=\widetilde{c}_{S}(k)+\widetilde{c}_{S}(k) \rho_{t} \widetilde{h}_{S}(k), \\
& \widetilde{h}_{D}(k)=\widetilde{c}_{D}(k)+\widetilde{c}_{D}(k) \rho_{t} \widetilde{h}_{D}(k),
\end{aligned}
$$


where $\rho_{t}=\rho_{1}+\rho_{2}$ is the total number density of ions in the solution (for the restricted electrolyte systems discussed here $\rho_{1}=\rho_{2}$ applies). We recall that the sum/difference decoupling suggested by equations (9) and (10) is only apparent, as the set of functions $\left\{h_{S}, c_{S}\right\}$ and $\left\{h_{D}, c_{D}\right\}$ are still entangled by the relations $(7)$ and (8).

\subsection{HNC closure}

In the set of equations (7)-(10), which provide the means for the calculation of the structure functions of the electrolyte solution, we still have to specify the sum and difference bridge functions $b_{S}(r)$ and $b_{D}(r)$. To do so is one of the goals of this study. In this section, however, we discuss the closure relations that result from the simplest possible approximation for these functions, namely when both bridge functions can be ignored (i. e., $b_{S}(r)=b_{D}(r)=0$ ). This is the well-known Hypernetted Chain (HNC) approximation, for which equations (4) and (5) simplify to

$$
\begin{aligned}
& h_{S}(r)=\mathrm{e}^{-\beta \bar{u}^{*}(r)+t_{S}(r)} \cosh \mathcal{D}_{0}(r)-1, \\
& h_{D}(r)=\mathrm{e}^{-\beta \bar{u}^{*}(r)+t_{S}(r)} \sinh \mathcal{D}_{0}(r),
\end{aligned}
$$

while the auxiliary function $\mathcal{D}(r)$ [cf. equation (6)] is given by

$$
\mathcal{D}_{0}(r)=-\beta \bar{u}_{D}(r)+t_{D}(r)
$$

It is interesting to analyze here a shortcoming of the HNC closure that is not widely appreciated. This may be seen most straightforwardly from equations (11) and (12). These equations can be formally solved for the function $\mathcal{D}_{0}(r)$ :

$$
\mathcal{D}_{0}(r)=\operatorname{arctanh}\{\chi(r)\}
$$

in terms of the auxiliary function

$$
\chi(r) \equiv \frac{h_{D}(r)}{1+h_{S}(r)} .
$$

Equation (14) for $\mathcal{D}_{0}(r)$ can then be substituted back into equation (11). Recalling that in the resulting equation $t_{S}(r)=h_{S}(r)-c_{S}(r)$ in the argument of the exponential, we can then solve for the sum combination $c_{S}(r)$ of the ion-ion direct correlation functions. We obtain, for values of $r$ large enough that we can make the approximation $\exp \left(-\beta \bar{u}^{*}(r)\right) \simeq 1$,

$$
c_{S}(r)=\ln \left\{\frac{\mathrm{e}^{h_{S}(r)}}{1+h_{S}(r)}\right\}-\frac{1}{2} \ln \left\{1-[\chi(r)]^{2}\right\} .
$$

The particular form of the second term in this equation follows straightforwardly from the identity $\cosh \operatorname{arctanh}(x)=1 / \sqrt{\left(1-x^{2}\right)}$. 
The feature, revealed by equation (16), that $c_{S}(r)$ under the HNC closure is expressed by a sum of two separate contributions, one that depends only on $h_{S}(r)$ while the other depends only on $\chi(r)$, is the source of difficulties when the closure is used to examine the material stability limit of the electrolyte solution. We recall [8] that the stability of the solution against phase separation (unmixing) is determined exclusively by the sum combination $c_{S}(r)$ of the direct correlation functions. (Briefly, the solution is stable or metastable against phase separation as long as $1-\rho_{t} \widetilde{c}_{S}(0) \geqslant$ 0 , where $\widetilde{c}_{S}(0)$ denotes the Fourier transform of $c_{S}(r)$ specialized at $\left.k=0\right)$. On the other hand, the signature of the states on the spinodal line (and, of course, at the critical point) is that at those thermodynamic states the sum combination $h_{S}(r)$ becomes infinitely long ranged (or, equivalently, $\widetilde{h}_{S}(k)$ diverges as $k \rightarrow 0$ at these states). In contrast, the range of $\chi(r)$, which is proportional to the difference combination $h_{D}(r)$, remains finite at the stability limit of the electrolyte solution [9].

At large values of the interionic distance $r$, where both $h_{S}(r)$ and $\chi(r)$ are small, we can approximate $c_{S}(r)$ in equation (16) by the first few terms of its power series expansion with respect to $h_{S}(r)$ and $\chi(r)$ :

$$
\begin{aligned}
c_{S}(r)= & \frac{1}{2}\left[h_{S}(r)\right]^{2}-\frac{1}{3}\left[h_{S}(r)\right]^{3}+\cdots \\
& +\frac{1}{2}[\chi(r)]^{2}+\frac{1}{4}[\chi(r)]^{4}+\cdots
\end{aligned}
$$

When the thermodynamic state approaches the stability limit and, correspondingly, $h_{S}(r)$ becomes more and more long-ranged, equation (17) reveals that, under the HNC closure, the sum combination $c_{S}(r)$ (due to the presence of terms with "pure" $\left[h_{S}(r)\right]^{n}$ powers) also becomes infinitely long-ranged .

\section{2. $\mathrm{HNC} / \mathrm{PY}$ closure}

As a first step in designing an integral equation relation that improves over the HNC closure, it is natural to introduce into the closure a bridge function $b_{S}(r)$ that eliminates from $c_{S}(r)$ the terms that contain pure $\left[h_{S}(r)\right]^{n}$ powers of the sum combination function $h_{S}(r)$. This can be accomplished most simply by choosing $b_{S}(r)$ of the form [7]

$$
b_{S}(r)=\ln \left[1+t_{S}(r)\right]-t_{S}(r) .
$$

We still insist on the approximation $b_{D}(r)=0$. When these approximations are introduced into the general equations (4) and (5) they become

$$
\begin{aligned}
& h_{S}(r)=\mathrm{e}^{-\beta \bar{u}^{*}(r)}\left[1+t_{S}(r)\right] \cosh \mathcal{D}_{0}(r)-1, \\
& h_{D}(r)=\mathrm{e}^{-\beta \bar{u}^{*}(r)}\left[1+t_{S}(r)\right] \sinh \mathcal{D}_{0}(r),
\end{aligned}
$$

with $\mathcal{D}_{0}(r)$ given by equation (13). 
In the next section we show that the choice of equation (18) for the sum combination $b_{S}(r)$ bridge function makes $c_{S}(r)$ under this closure short-ranged (which is the correct behaviour) at the critical point or any other state along the spinodal line of the electrolyte solution.

Equation (18) is the well-known form that the bridge function for a pure substance takes under the Percus-Yevick (PY) integral equation closure. In fact, the PY flavour of this closure becomes immediately obvious in equation (19) if we ignore the charge-fluctuation factor $\cosh \mathcal{D}_{0}(r)$. On the other hand, through the choice $b_{D}(r)=0$ for the difference bridge function combination, this closure retains all the features of the HNC closure in what concerns the difference combination direct correlation function $c_{D}(r)$. For these reasons we use the acronym HNC/PY to refer to this closure. A version of this closure for general models (i. e., not restricted) of electrolyte solutions has been reported [7].

As the calculations reported in section 3 show, the performance of the HNC/PY closure for dilute solutions of 2-2 restricted electrolytes is somewhat disappointing, with the HNC closure giving (broadly speaking) better results. The goal of this paper is, using the HNC/PY closure as the foundation, to propose a new family of improved integral equation closures for solutions of symmetric electrolytes.

\subsection{Beyond the PY form for the sum bridge function $b_{S}(r)$}

To obtain improved results but, at the same time to preserve the feature that $c_{S}(r)$ is of finite range at every thermodynamic state, we slightly modify the PY form of the sum combination $b_{S}(r)$ of the bridge functions [cf. equation (18)]. We do this by adding a corrector function $\lambda(r)$ to the argument of the logarithmic term:

$$
b_{S}(r)=\ln \left[1+t_{S}(r)+\lambda(r)\right]-t_{S}(r) .
$$

For generality we do not assume at this point that $b_{D}(r)=0$, but instead we leave this function momentarily unspecified.

The general equations (4) and (5) take the form

$$
\begin{aligned}
& h_{S}(r)=\mathrm{e}^{-\beta \bar{u}^{*}(r)}\left[1+t_{S}(r)+\lambda(r)\right] \cosh \mathcal{D}(r)-1, \\
& h_{D}(r)=\mathrm{e}^{-\beta \bar{u}^{*}(r)}\left[1+t_{S}(r)+\lambda(r)\right] \sinh \mathcal{D}(r),
\end{aligned}
$$

where $\mathcal{D}(r)$ was introduced in equation (6).

We follow now the discussion in section 2.1. We begin by solving equations (22) and (23) for the auxiliary function $\mathcal{D}(r)$ :

$$
\mathcal{D}(r)=\operatorname{arctanh}[\chi(r)],
$$

where $\chi(r)$ is given in equation (15).

At interionic distances such that $\exp \left(-\beta \bar{u}^{*}(r)\right) \simeq 1$, equations $(22)$ and $(24)$ may be solved for the sum combination $c_{S}(r)$ of direct correlation functions, with the result

$$
c_{S}(r)=\lambda(r)+\left(1+h_{S}(r)\right)\left\{1-\sqrt{1-[\chi(r)]^{2}}\right\} .
$$


To derive this equation we have used the identity

$$
\operatorname{sech} \operatorname{arctanh}[\chi(r)]=\sqrt{1-[\chi(r)]^{2}} .
$$

Equation (25) shows that the contribution $\lambda(r)$ coming from the sum bridge function $b_{S}(r)$ is separated from the remaining terms. Except for the multiplicative factor $\left(1+h_{S}(r)\right)$, these terms depend only on the function $\chi_{D}(r)$, which is shortranged at every thermodynamic state. Furthermore, the equation does not explicitly involve the bridge function $b_{D}(r)$ : this expression of $c_{S}(r)$ in terms of $\lambda(r), h_{S}(r)$ and $\chi(r)$ is not affected by whichever approximation we choose for $b_{D}(r)$.

At large values of $r$, where $\chi(r)$ is small, we can approximate equation (25) for $c_{S}(r)$ by

$$
c_{S}(r)=\lambda(r)+h_{D}(r)\left\{\frac{1}{2} \chi(r)+\frac{1}{8}[\chi(r)]^{3}+\cdots\right\},
$$

in which $h_{S}(r)$ does not appear explicitly

Leaving aside for a moment the function $\lambda(r)$, equation (26) shows that at large $r$ the sum combination $c_{S}(r)$ involves terms from which pure powers $\left[h_{S}(r)\right]^{n}$ are absent; such powers of $h_{S}(r)$ (that originate from the expansion of the denominator of $\chi(r))$ are always multiplied by powers $\left[h_{D}(r)\right]^{m}$ of the difference combination function $h_{D}(r)$. The latter function is of finite range at every thermodynamic state [9]. When $\lambda(r)=0$, equation (26) coincides with the large- $r$ behaviour of $c_{S}(r)$ under the HNC/PY closure (cf. section 2.2). This demonstrates the statement made in that section that $c_{S}(r)$ under the HNC/PY closure is of finite-range at the stability limit of the ionic solution.

In view of the extreme complexity involved in the direct calculation of $\lambda(r)$, here we simply consider this function to be a functional of the functions $\chi(r)$ and $h_{D}(r)$ (or, equivalently, given equation (15), a functional of $h_{S}(r)$ and $h_{D}(r)$ ). Furthermore, we propose that this functional has a form that closely resembles the first terms in the asymptotic expansion of $c_{S}(r)$ under the HNC/PY closure [which is given by equation (26) after making $\lambda(r)=0]$. With these assumptions it follows that the simplest candidate for $\lambda(r)$ has the form

$$
\lambda(r)=\frac{1}{2} B_{S} h_{D}(r) \chi(r),
$$

where the value of the bridge parameter $B_{S}$ needs to be specified.

Replacing this form of $\lambda(r)$ back into equation (26) gives the asymptotic behaviour of the sum direct correlation function $c_{S}(r)$ at large $r$

$$
c_{S}(r)=\left(A_{S} / 2\right) h_{D}(r) \chi(r)+(1 / 8) h_{D}(r)[\chi(r)]^{3}+\cdots
$$

with the parameter $A_{S}$ is related to $B_{S}$ by $A_{S}=1+B_{S}$.

It is clear that this approach for constructing $\lambda(r)$, that borrows from the asymptotic expansion of $c_{S}(r)$ under HNC/PY, guarantees that $c_{S}(r)$ under the new closure [that incorporates $\lambda(r)$ ] remains of finite range at the stability limit of the electrolyte solution. It is also worth noting that the strategy just outlined for the construction of $\lambda(r)$ is completely independent on which approximation we choose for the difference bridge function $b_{D}(r)$. 


\subsection{Approximation for the difference bridge function $b_{D}(r)$}

As in the case of $\lambda(r)$, we propose to construct an approximation for the difference bridge function $b_{D}(r)$ by considering the first few terms of the asymptotic form of $c_{D}(r)$ under the HNC/PY approximation.

From equations (6) and (24) we find for $c_{D}^{*}(r) \equiv c_{D}(r)+\beta \bar{u}_{D}(r)$ (the short-range part of the difference direct correlation function)

$$
c_{D}^{*}(r)=b_{D}(r)+h_{D}(r)-\frac{1}{2} \ln \left\{\frac{1+\chi(r)}{1-\chi(r)}\right\} .
$$

The last term is, of course, equivalent to $\operatorname{arctanh}\{\chi(r)\}$. This expression is explicitly linear in $h_{D}(r)$. Expanding the logarithmic term in powers of $\chi(r)$ we obtain

$$
c_{D}^{*}(r)=b_{D}(r)+h_{D}(r)-\chi(r)-\frac{1}{3}[\chi(r)]^{3}-\frac{1}{5}[\chi(r)]^{5}+\cdots .
$$

The second and third terms can be combined together, taking into account equation (15), so that the large- $r$ asymptotic expansion of $c_{D}^{*}(r)$ becomes

$$
c_{D}^{*}(r)=b_{D}(r)+h_{S}(r) \chi(r)-\frac{1}{3}[\chi(r)]^{3}+\cdots .
$$

It is important to note that this result applies irrespective of any approximation made to $b_{S}(r)$.

By taking $b_{D}(r)=0$ in equation (31) we obtain the asymptotic expansion of $c_{D}(r)$ under any closure that approximates the difference bridge function to zero, like the HNC or HNC/PY closures. We may generate an approximate expression for $b_{D}(r)$ by proceeding as we did for constructing $\lambda(r)$ in the last section; i. e., we assume that $b_{D}(r)$ is proportional to the first term of the asymptotic expansion of $c_{D}^{*}(r)$ under the HNC/PY closure. We then have

$$
b_{D}(r)=B_{D} h_{S}(r) \chi(r),
$$

in terms of the bridge parameter $B_{D}$. With this approximation $c_{D}^{*}(r)$ [equation (31)] behaves at large $r$ as

$$
c_{D}^{*}(r)=A_{D} h_{S}(r) \chi(r)-\frac{1}{3}[\chi(r)]^{3}+\cdots,
$$

where $A_{D}=1+B_{D}$. Clearly, this expansion does not contain terms with pure powers $\left[h_{S}(r)\right]^{n}$; such powers appear to be always multiplied by powers $\left[h_{D}(r)\right]^{m}$ that are of finite range for every thermodynamic state.

Finally, it is straightforward to show from equations (28) and (33), together with the expected small- $k$ behaviour of the Fourier transforms of $h_{S}(r)$ and $h_{D}(r)$

$$
\begin{aligned}
& \widetilde{h}_{S}(k)=k^{0} h_{S}^{(0)}+k^{2} h_{S}^{(2)}+O\left(k^{4}\right), \\
& \widetilde{h}_{D}(k)=k^{0} h_{D}^{(0)}+k^{2} h_{D}^{(2)}+O\left(k^{4}\right),
\end{aligned}
$$

that the direct correlation functions $c_{S}(r)$ and $c_{D}(r)$ under the closures proposed in this section have the form that is required for the Stillinger-Lovett second moment condition $[10]$ to be satisfied. 


\section{Calculations}

\subsection{Closure relations}

The development in section 2 motivated specific forms for the sum $b_{S}(r)$ and difference $b_{D}(r)$ bridge functions [equations (21) and (27) for $b_{S}(r)$; equation (32) for $\left.b_{D}(r)\right]$. Having this information, we may think of several approximate implementations of equations (7) and (8):

HNC closure. In this closure we have $b_{S}(r)=b_{D}(r)=0$ at every $r$. The sum and difference direct correlation functions $c_{S}(r)$ and $c_{D}(r)$ are given by relations similar to equations (7) and (8), but with $b_{S}(r)$ absent from the argument of the exponential, and with $\mathcal{D}_{0}(r)$ in place of $\mathcal{D}(r)$ [cf. equations (13) and (6)].

HNC/PY closure. In this closure $\lambda(r)=b_{D}(r)=0$ at every $r$. Hence $c_{S}(r)$ and $c_{D}(r)$ are given by expressions similar to equations (7) and (8), but with $b_{S}(r)$ given by equation (18) and, again, with $\mathcal{D}_{0}(r)$ in place of $\mathcal{D}(r)$.

$\mathrm{HNC} / \lambda_{1}$ closure. In this closure $b_{S}(r)$ in the argument of the exponentials in equations (7) and (8) is given by equation (21), with $\lambda(r)$ represented by (27). The auxiliary function $\mathcal{D}(r)$ again takes the form of $\mathcal{D}_{0}(r)$ [i. e., in this closure we again assume that $b_{D}(r)=0$ at every $\left.r\right]$.

$b_{D} / \lambda_{1}$ closure. In this closure $c_{S}(r)$ and $c_{D}(r)$ take the form of equations $(7)$ and (8), with $b_{S}(r)$ given by equation $(21), \lambda(r)$ given by equation $(27)$, and $b_{D}(r)$ given by equation (32).

It is obvious that the implementation of closures $\mathrm{HNC} / \lambda_{1}$ and $b_{D} / \lambda_{1}$ require the specification of the bridge parameters $B_{S}$ and $B_{D}$. We address this important issue in the case of the $\mathrm{HNC} / \lambda_{1}$ closure in the next subsection.

In what remains of section 3 we report numerical results for the structure and thermodynamic properties of dilute solutions of a model 2-2 electrolyte as calculated with the HNC, HNC/PY, and HNC/ $\lambda_{1}$ closures. We briefly comment on closure $b_{D} / \lambda_{1}$ in section 4 .

\subsection{Numerical algorithm, electrolyte model, and calculations}

To solve the Ornstein-Zernike integral equations [cf. equations (9) and (10)] under any of the above closure relations we have used the familiar Picard iterative approach. The technical difficulties associated with the long-range nature of the difference direct correlation function $c_{D}(r)$ are avoided by means of Ng's method $[11,12]$.

As a representative of a restricted model of an electrolyte solution, we consider the model of a 2-2 electrolyte studied by Duh and Haymet [6]. In this model the ions interact through a McMillan-Mayer level potential of the form of equation (2), with $\bar{u}^{*}(r)$ given by

$$
\bar{u}^{*}(r)=\frac{k_{\mathrm{B}} B}{\sigma}\left(\frac{\sigma}{r}\right)^{9}
$$


where $B=5377(z e)^{2} \AA \mathrm{K}$ and $\sigma=2.8428 \AA$. In the Coulombic term $\bar{u}_{D}(r)$ we specify $z=2$ and $\epsilon_{0}=78.358$, the value of the dielectric constant of water at $298.15 \mathrm{~K}$.

The calculations reported here for this model electrolyte parallel those in the study by Duh and Haymet [6]. We have solved each of the integral equation closures summarized in the previous section for electrolyte solutions at several concentrations in the range $0.001 \mathrm{~mol} \mathrm{dm}^{-3} \leqslant c_{s} \leqslant 0.5625 \mathrm{~mol} \mathrm{dm}^{-3}$, where $c_{s}$ is the molar concentration of the electrolyte.

In addition to the structure functions [the cation-cation $g_{11}(r)$ and the cationanion $g_{12}$ radial distribution functions, obtained from $g_{S}(r)$ and $h_{D}(r)$ by inversion of equations (3)], we have calculated the following thermodynamic properties:

(a) the reduced excess internal energy per ion $u \equiv \beta U^{\mathrm{ex}} / N_{t}$ :

$$
u=2 \pi \beta \rho_{t}\left\{\int_{0}^{\infty} \mathrm{d} r r^{2} \bar{u}^{*}(r) g_{S}(r)+\frac{z^{2} e^{2}}{\epsilon_{0}} \int_{0}^{\infty} \mathrm{d} r r h_{D}(r)\right\} .
$$

(b) the osmotic coefficient $\phi \equiv \beta \pi / \rho_{t}$ of the solution:

$$
\phi=1-\frac{2 \pi \beta \rho_{t}}{3}\left\{\int_{0}^{\infty} \mathrm{d} r r^{3} \frac{\mathrm{d} \bar{u}^{*}(r)}{\mathrm{d} r} g_{S}(r)-\frac{z^{2} e^{2}}{\epsilon_{0}} \int_{0}^{\infty} \mathrm{d} r r h_{D}(r)\right\} .
$$

(c) the generalized compressibility:

$$
\beta\left(\frac{\partial \pi}{\partial \rho_{t}}\right)_{T}=1-4 \pi \rho_{t} \int_{0}^{\infty} \mathrm{d} r r^{2} c_{S}(r) .
$$

In the above equations $\pi$ is the osmotic pressure of the solution. It may be shown that the generalized compressibility equals

$$
\beta\left(\frac{\partial \pi}{\partial \rho_{t}}\right)_{T}=\beta \rho_{s}\left(\frac{\partial \mu_{s}}{\partial \rho_{t}}\right)_{T}=1+\left(\frac{\partial \ln \gamma_{ \pm}}{\partial \ln \rho_{s}}\right)_{T}
$$

where $\mu_{s}$ and $\gamma_{ \pm}$are, respectively, the chemical potential and the mean activity coefficient of the electrolyte, while $\rho_{s}$ is the number density of the salt $\left(\rho_{s}=\rho_{t} / \nu\right.$, where $\nu=\nu_{1}+\nu_{2}$ is the sum of the cation and anion stoichiometric coefficients).

It is important to remember that equations (37)-(39) correspond to different thermodynamic routes [energy route for $u$, virial route for $\phi$, and compressibility route for $\left.\beta\left(\partial \pi / \partial \rho_{t}\right)_{T}\right]$, and when implemented using correlation functions $g_{S}(r)$ and $h_{D}(r)$ calculated from approximate integral equation closures, their results are not necessarily thermodinamically consistent.

\subsection{Results}

As a representative of the results obtained at each concentration examined, we first discuss in some detail the results obtained under the HNC, HNC/PY, and $\mathrm{HNC} / \lambda_{1}$ closures for the model electrolyte solution at concentration $c_{s}=0.02 \mathrm{~mol}$ $\mathrm{dm}^{-3}$ at temperature $298.15 \mathrm{~K}$. 

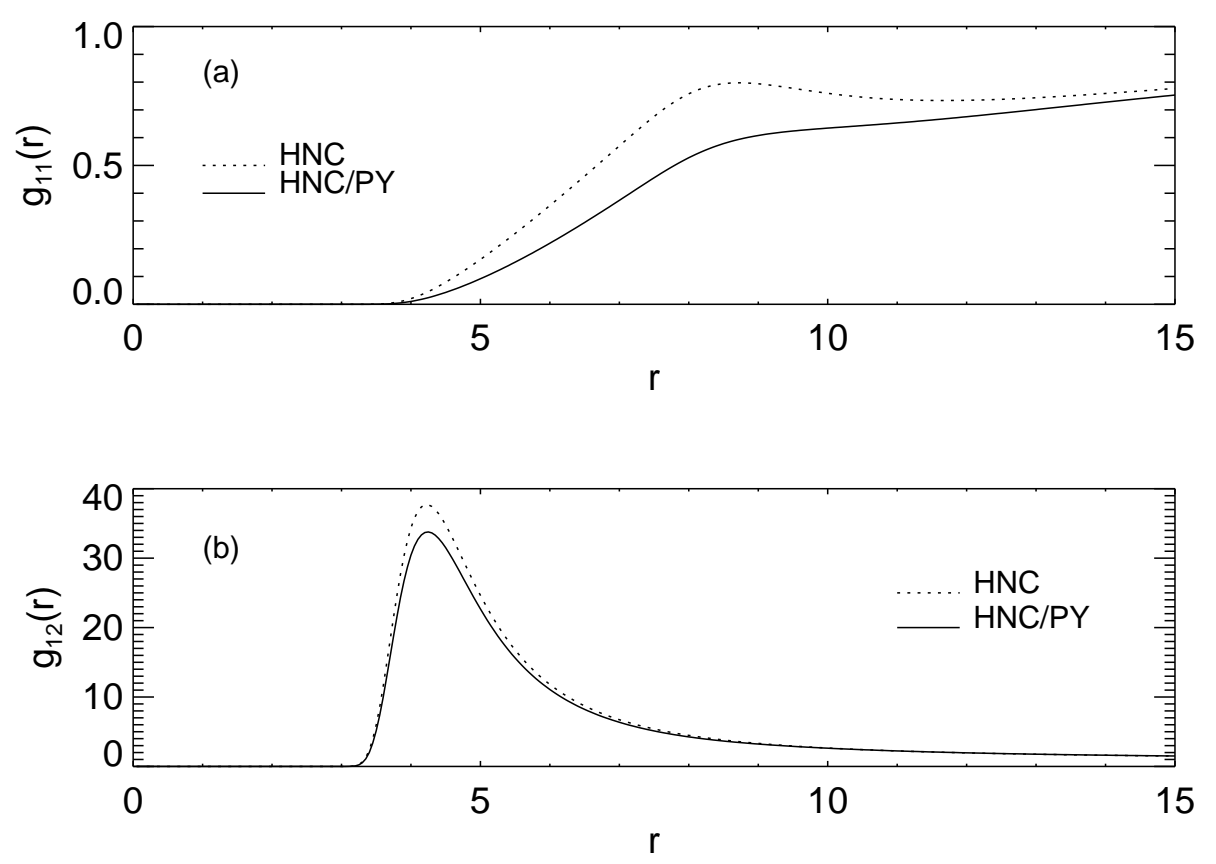

Figure 1. Ion-ion radial distribution functions for an aqueous solution of a restricted 2-2 electrolyte at $c_{s}=0.02 \mathrm{~mol} \mathrm{dm}^{-3}$ and $298.15 \mathrm{~K}$. The correlation function are calculated under the integral equation closures HNC and HNC/PY. (a): Like-sign (cation-cation or anion-anion) correlation function $g_{11}(r)$; (b): cationanion correlation function $g_{12}(r) . r$ is in units of $\AA$.

In figure 1 we report the pair correlation functions $g_{11}(r)$ and $g_{12}(r)$ at this concentration as calculated under the $\mathrm{HNC}$ and the $\mathrm{HNC} / \mathrm{PY}$ integral equation closures. The first thing to notice is that $\left(g_{12}^{\max }\right)_{\mathrm{MD}}=48.0$, the peak height for the cation-anion correlation function $g_{12}(r)$ calculated from molecular dynamics computer simulation [5], is substantially larger than the corresponding $\mathrm{HNC}$ and $\mathrm{HNC} / \mathrm{PY}$ results, $\left(g_{12}^{\max }\right)_{\mathrm{HNC}}=37.6$ and $\left(g_{12}^{\max }\right)_{\mathrm{HNC} / \mathrm{PY}}=33.7$. From this perspective, and in spite of the fact that for this closure $b_{S}(r) \neq 0$, the HNC/PY closure does not improve over the HNC predictions. We notice, however, that there is no evidence of the spurious peak at $r \simeq 8.5 \AA$ in the like-sign ion-ion correlation function $g_{11}(r)$ calculated under the HNC/PY closure, contrary to the case for $g_{11}(r)$ under HNC closure. The simulation study [5] indicates that such a peak is an artifact of the approximate $\mathrm{HNC}$ closure.

With respect to the thermodynamic properties at this concentration, we find $u_{\mathrm{HNC} / \mathrm{PY}}=-1.245$ and $\phi_{\mathrm{HNC} / \mathrm{PY}}=0.709$, which should be compared with the HNC results $u_{\mathrm{HNC}}=-1.280$ and $\phi_{\mathrm{HNC}}=0.713$ and with the molecular dynamics results $[5,6] u_{\mathrm{MD}}=-1.413$ and $\phi_{\mathrm{MD}}=0.706$.

Thus, except for the case of the osmotic coefficient and the absence of the peak in $g_{11}(r)$, at this concentration of the 2-2 electrolyte the structure and thermodynamic properties obtained with the $\mathrm{HNC} / \mathrm{PY}$ closure differ from the simulation results by a larger extent than the predictions of the HNC closure. This pattern in the performance comparison between $\mathrm{HNC}$ and $\mathrm{HNC} / \mathrm{PY}$ is also observed, in different 

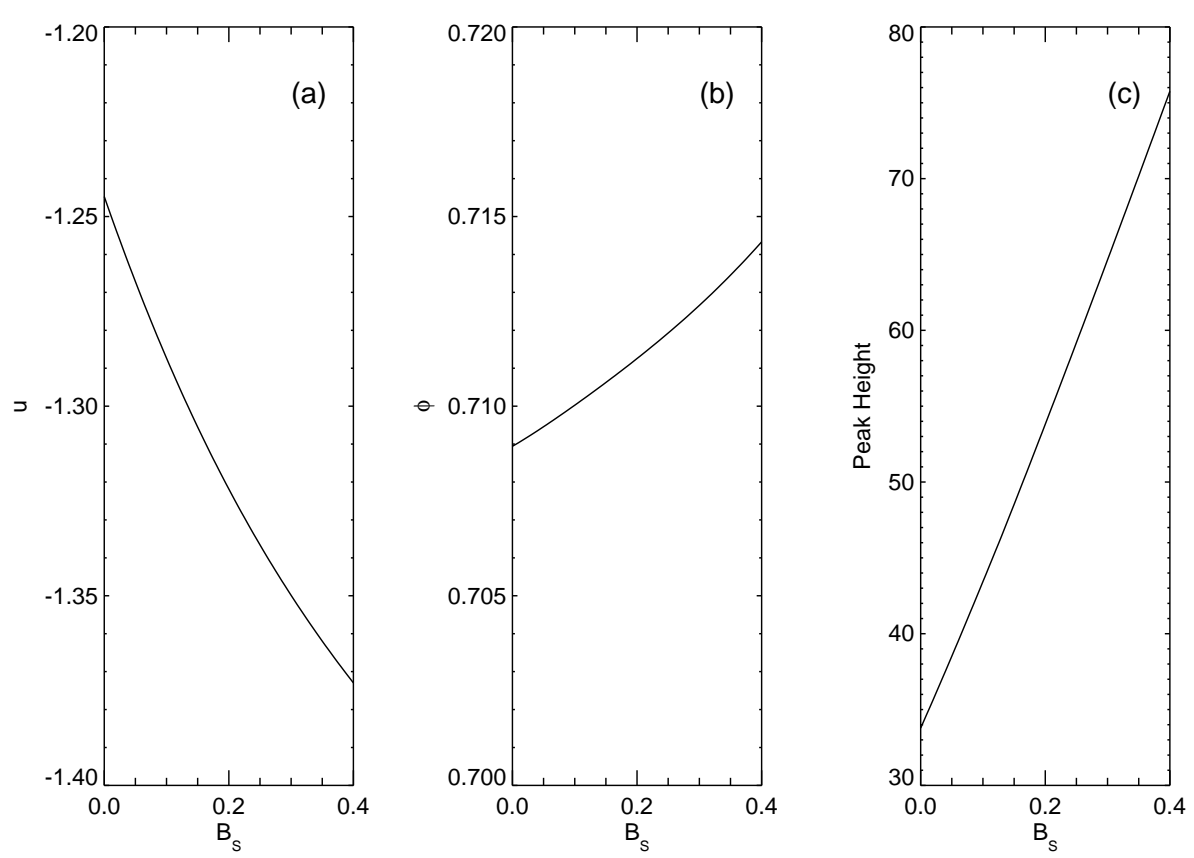

Figure 2. Dependence of thermodynamic and structural properties of an electrolyte solution with the bridge parameter $B_{S}$ under closure $\mathrm{HNC} / \lambda_{1}$. The results shown correspond to a $2-2$ aqueous electrolyte solution of concentration $c_{s}=0.02 \mathrm{~mol} \mathrm{dm}^{-3}$ at $298.15 \mathrm{~K}$. (a) Reduced excess internal energy per ion, $u$, calculated with equation (37); (b) osmotic coefficient $\phi$, calculated with equation (38); (c) peak height $g_{12}^{\max }$ in the cation-anion pair correlation function $g_{12}(r)$.

degrees, at the other electrolyte concentrations. The differences between the two closures disappear for concentrations above $c_{s} \simeq 0.5 \mathrm{~mol} \mathrm{dm}^{-3}$.

We now turn to the $\mathrm{HNC} / \lambda_{1}$ integral equation closure. The HNC/PY closure discussed above is a particular case of $\mathrm{HNC} / \lambda_{1}$, and is obtained when we set to zero the bridge parameter $B_{S}$ in equation (27). The complete specification of the $\mathrm{HNC} / \lambda_{1}$ closure requires that we select the value of $B_{S}$.

In figure 2 we illustrate the dependence of $u, \phi$, and $g_{12}^{\max }$ with $B_{S}$ at $c_{s}=0.02 \mathrm{~mol}$ $\mathrm{dm}^{-3}$ and $298.15 \mathrm{~K}$. We observe that both $u$ and $g_{12}^{\max }$ are quite sensitive to the value of $B_{S}$, and that as we increase the value of this parameter both $u$ and $g_{12}^{\max }$ change, respectively, to more negative and more positive values, thus improving the predictions of the closure when compared with the molecular dynamics data. On the other hand, $\phi_{\mathrm{HNC} / \mathrm{PY}}$ is already in good agreement with $\phi_{\mathrm{MD}}$, and as $B_{S}$ is increased the agreement of $\phi$ under this closure deteriorates. It should be noted, however, that the dependence of $\phi$ with $B_{S}$ is much milder than the dependence of the other properties.

More careful examination of the figure reveals $u$ and $g_{12}^{\max }$ change with $B_{S}$ towards the target values (respectively $u_{\mathrm{MD}}$ and $\left.\left(g_{12}^{\max }\right)_{\mathrm{MD}}\right)$ at different rates. For example, at $B_{S}=0.14$ we find that $\left(g_{12}^{\max }\right)_{\mathrm{HNC} / \lambda_{1}}=47.49$, which approximately matches the molecular dynamics result $\left(g_{12}^{\max }\right)_{\mathrm{MD}}=48.0$. However, at this particular value of $B_{S}$ we find $u_{\mathrm{HNC} / \lambda_{1}}=-1.302$ which, although an improvement over both the HNC/PY 

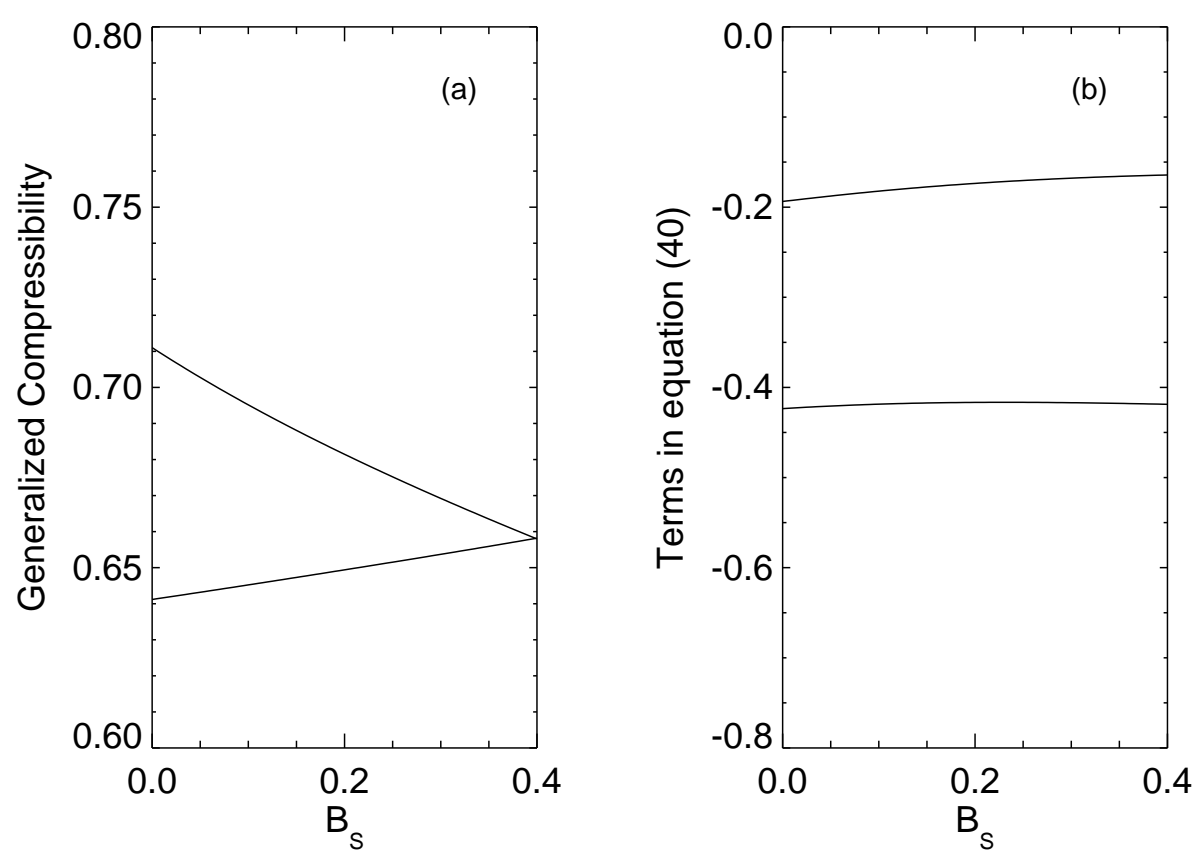

Figure 3. Sensitivity of two thermodynamic consistency tests with the bridge parameter $B_{S}$ in the $\mathrm{HNC} / \lambda_{1}$ integral equation closure. The results shown correspond to a 2-2 aqueous electrolyte solution of concentration $c_{s}=0.02 \mathrm{~mol}$ $\mathrm{dm}^{-3}$ at $298.15 \mathrm{~K}$. (a) Generalized compressibility test: $\beta\left(\partial \pi / \partial \rho_{t}\right)_{T}$ calculated according to the compressibility route (upper curve) and the virial route (lower curve). (b) Maxwel relation derived test: (energy route)-based left hand side of equation (42) (lower curve) and (compressibility route)-based right hand side of equation (42) (upper curve).

and $\mathrm{HNC}$ values, respectively $u_{\mathrm{HNC} / \mathrm{PY}}=-1.245$ and $u_{\mathrm{HNC}}=-1.280$, differs from the considerably more negative molecular dynamics result $u_{\mathrm{MD}}=-1.413$. With regard to the osmotic coefficient, we find $\phi_{\mathrm{HNC} / \lambda_{1}}=0.7105$ at $B_{S}=0.14$, which should be compared with the simulation result $\phi_{\mathrm{MD}}=0.706$. The $\mathrm{HNC} / \lambda_{1}$ result is slightly better than the HNC prediction, $\phi_{\mathrm{HNC}}=0.713$, but as mentioned above, $\phi_{\mathrm{HNC} / \mathrm{PY}}=0.709$ is in a better agreement with simulation. Thus, at $B_{S}=0.14$, when $\left(g_{12}^{\max }\right)_{\mathrm{HNC} / \lambda_{1}} \simeq\left(g_{12}^{\max }\right)_{\mathrm{MD}}$, the reduced excess energy per ion $u_{\mathrm{HNC} / \lambda_{1}}$ betters $u_{\mathrm{HNC}}$, but it is still far from the target $u_{\mathrm{MD}}$.

Conversely, we observe from figure $2 \mathrm{a}$ that at $B_{S}=0.4$ the reduced excess energy is $u_{\mathrm{HNC} / \lambda_{1}}=-1.372$, which is still short of matching $u_{\mathrm{MD}}=-1.413$. At this value of $B_{S}$, however, $\left(g_{12}^{\max }\right)_{\mathrm{HNC} / \lambda_{1}}=76$ is far too large when compared with the $\left(g_{12}^{\max }\right)_{\mathrm{MD}}=48.0$.

To further illustrate the dependence with $B_{S}$ of the thermodynamic properties calculated under the $\mathrm{HNC} / \lambda_{1}$ closure, figure 3 reports two independent consistency tests for properties calculated according to different thermodynamic routes.

One test [figure 3a] addresses the thermodynamic consistency for the generalized compressibility $\beta\left(\partial \pi / \partial \rho_{t}\right)_{T}$ under the $\mathrm{HNC} / \lambda_{1}$ closure when this property is calculated using either the compressibility route [equation (39)] or the virial route. The 
latter route involves numerical computation of the indicated partial derivative, with the function $\beta \pi$ at each density calculated with the relation $\beta \pi=\rho_{t} \phi$, in which the osmotic coefficient is obtained with the help of the (virial route)-based equation (38).

The other thermodynamic consistency test considered follows from the Maxwell relation between the reduced internal energy and the reduced chemical potential of the electrolyte [8]:

$$
\nu\left(\frac{\partial\left(\rho_{t}[3 / 2+u]\right)}{\partial \rho_{t}}\right)_{\beta}=\left(\frac{\partial\left(\beta \mu_{s}\right)}{\partial \beta}\right)_{\rho_{t}} .
$$

Notice that the left hand side of the relation requires the full reduced internal energy per ion; the term $3 / 2$ is just the value of the non-interacting contribution $\beta U^{\text {id }} / N_{t}$ to the reduced internal energy. By taking the derivative of each side of equation (41) with respect to the total ionic density $\rho_{t}$ we derive, after taking into account the first equality of equation (40),

$$
\rho_{t}^{2} \frac{\partial^{2} u}{\partial \rho_{t}^{2}}+2 \rho_{t} \frac{\partial u}{\partial \rho_{t}}=-T \frac{\partial}{\partial T}\left\{\beta\left(\frac{\partial \pi}{\partial \rho_{t}}\right)_{T}\right\}
$$

It should be noted that the partial derivatives on the left hand side of this equation are carried at constant temperature, while those on the right hand side are carried at constant density of the ions. To use this equation as a measure of the thermodynamic consistency of closure $\mathrm{HNC} / \lambda_{1}$, we calculate each side of the equation following a different thermodynamic route. Specifically, for the left hand side we calculate $u$ at several values of $\rho_{t}$ using the energy route, equation (37). For the right hand side we calculate $\beta\left(\partial \pi / \partial \rho_{t}\right)_{T}$ at several temperatures using the compressibility route, equation (39). The results are shown in figure $3 \mathrm{~b}$.

Figure $3 \mathrm{a}$ shows that at $B_{S}=0$, which corresponds to the $\mathrm{HNC} / \mathrm{PY}$ closure, the difference between the values of the generalized compressibility $\beta\left(\partial \pi / \partial \rho_{t}\right)_{T}$ calculated under the compressibility and virial routes is the largest, with the value calculated by the compressibility route larger than the one obtained through the virial route. The thermodynamic inconsistency decreases, however, when $B_{S}$ is increased, and the figure shows that at $B_{S} \simeq 0.395$ the two routes in fact give the same value for $\beta\left(\partial \pi / \partial \rho_{t}\right)_{T}$. Although this is satisfying, this thermodynamic consistency is achieved at a value of $B_{S}$ where $g_{12}^{\max }$ is too large and, furthermore, for which the shape of $g_{11}(r)$ differs considerably from the like-sign correlation function extracted from molecular dynamics simulation (see below).

Figure $3 \mathrm{~b}$ illustrates the performance of the $\mathrm{HNC} / \lambda_{1}$ closure with respect to the second thermodynamic consistency test, equation (42). It is evident from the figure that under this closure the (energy route)-based left hand side of the relation is different at every $B_{S}$ from the (compressibility route)-based right hand side. Furthermore, the magnitude of the thermodynamic inconsistency is practically independent of the value of the bridge parameter $B_{S}$.

Finally, in figure 4 we report the ion-ion correlation functions $g_{11}(r)$ and $g_{12}(r)$ for the 2-2 electrolyte at concentration $c_{s}=0.02 \mathrm{~mol} \mathrm{dm}^{-3}$. In addition to the HNC and $\mathrm{HNC} / \mathrm{PY}$ results already shown in figure 1, we also display the correlation functions 

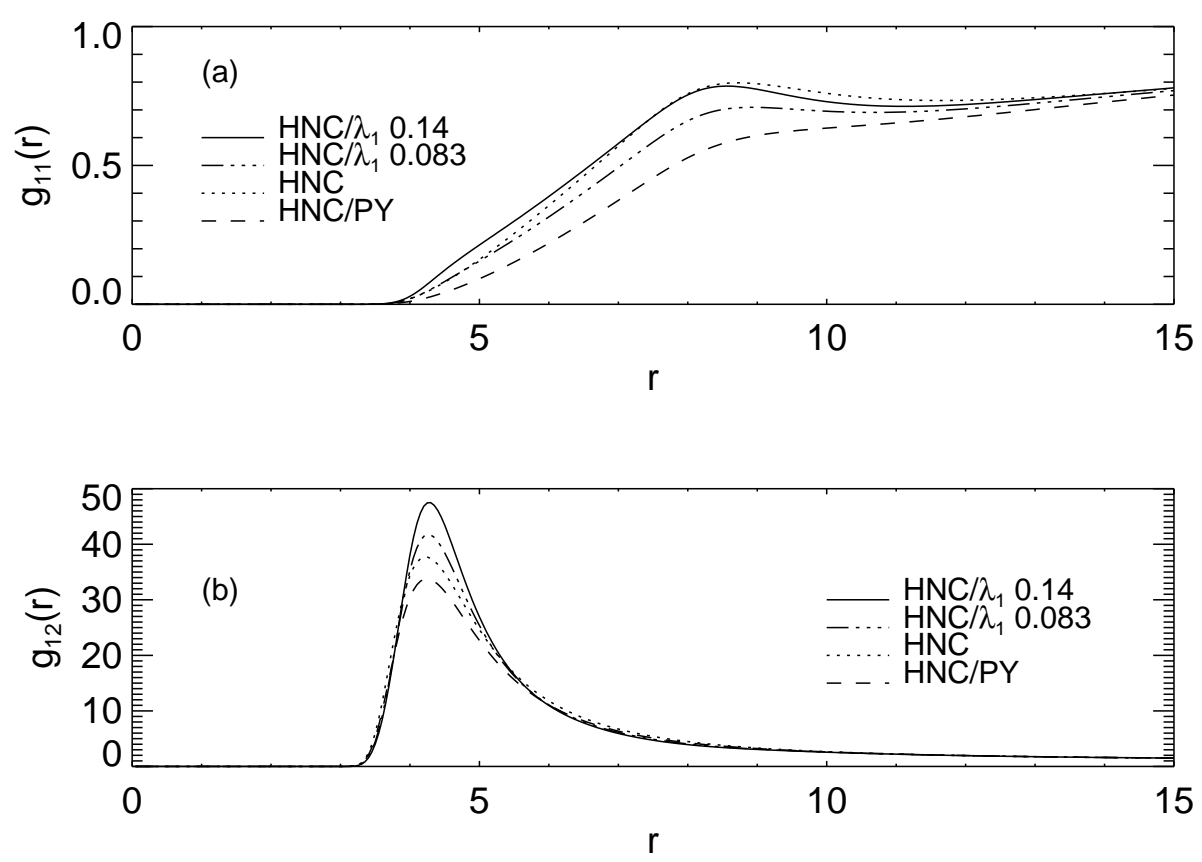

Figure 4. Ion-ion radial distribution functions for an aqueous solution of a restricted 2-2 electrolyte at $c_{s}=0.02 \mathrm{~mol} \mathrm{dm}^{-3}$ and $298.15 \mathrm{~K}$. The correlation function are calculated under the integral equation closures $\mathrm{HNC}, \mathrm{HNC} / \mathrm{PY}$, and $\mathrm{HNC} / \lambda_{1}$ for two values of the bridge parameter $B_{S}$. These are $B_{S}=0.14$, when $\left(g_{12}^{\max }\right)_{\mathrm{HNC} / \lambda_{1}}=\left(g_{12}^{\max }\right)_{\mathrm{HNC}}$, and $B_{S}=0.083$, when $u_{\mathrm{HNC} / \lambda_{1}}=u_{\mathrm{HNC}}$. (a): Likesign (cation-cation or anion-anion) correlation function $g_{11}(r)$; (b): cation-anion correlation function $g_{12}(r) . r$ is in units of $\AA$.

calculated under $\mathrm{HNC} / \lambda_{1}$ using two different choices, $B_{S}=0.14$ and $B_{S}=0.083$, of the bridge parameter.

As discussed above, when $B_{S}=0.14$ (at $c_{s}=0.02 \mathrm{~mol} \mathrm{dm}{ }^{-3}$ ) the $\mathrm{HNC} / \lambda_{1}$ closure gives a cation-anion correlation function $g_{12}(r)$ whose preak height $\left(g_{12}^{\max }\right)_{\mathrm{HNC} / \lambda_{1}}$ approximately equals $\left(g_{12}^{\max }\right)_{\mathrm{MD}}$, the value observed in the molecular dynamics simulation. Figure $4 \mathrm{~b}$ shows that for this value of $B_{S}$ the $\mathrm{HNC} / \lambda_{1}$ correlation function $g_{12}(r)$ compares very favourably with the corresponding molecular dynamics result $[5,6]$. (We refer the reader to references $[5,6]$ for the relevant figures of the correlation functions obtained by molecular dynamics). Unfortunately, figure 4a reveals that for this value of $B_{S}$ the like-sign correlation function $g_{11}(r)$ develops the same spurious peak at $r \simeq 8.5 \AA$ that plagues the HNC closure. We have found this same behaviour of closure $\mathrm{HNC} / \lambda_{1}$ at electrolyte concentrations $c_{s} \leqslant 0.02 \mathrm{~mol} \mathrm{dm}^{-3}$ : for values of $B_{S}$ such that $\left(g_{12}^{\max }\right)_{\mathrm{HNC} / \lambda_{1}} \simeq\left(g_{12}^{\max }\right)_{\mathrm{MD}}$ the shape of $g_{11}(r)$ under $\mathrm{HNC} / \lambda_{1}$ deteriorates to a level comparable to $g_{11}(r)$ under the HNC closure. For even larger values of $B_{S}$ [as would be needed for achieving compressibility-virial thermodynamic consistency in the computation of $\beta\left(\partial \pi / \partial \rho_{t}\right)_{T}$ ] the spurious peak is more intense, and the quality of the predicted structure of the solution is not acceptable.

The results reported so far eliminate the possibility of seeking thermodynamic consistency as a practical and unbiased approach for fixing the strength of the 

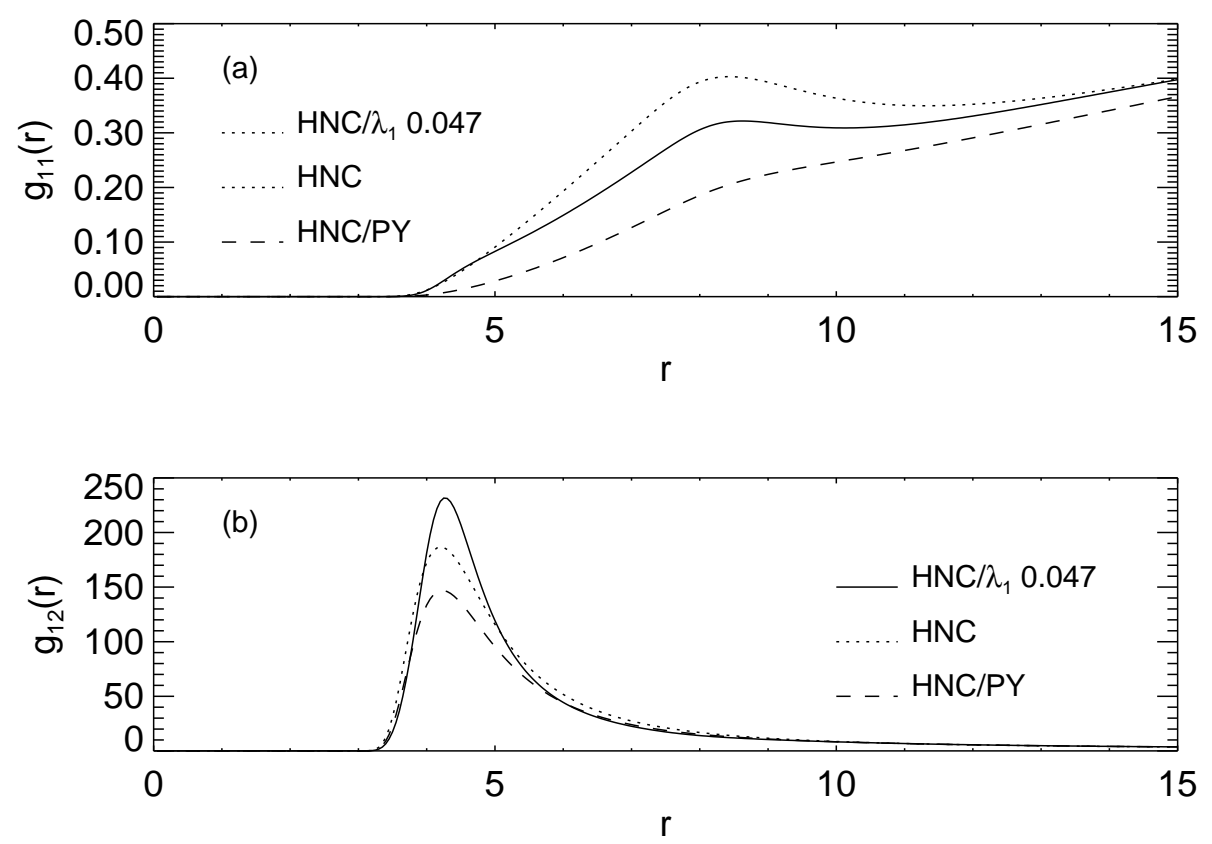

Figure 5. Ion-ion radial distribution functions for an aqueous solution of a restricted 2-2 electrolyte at $c_{s}=0.001 \mathrm{~mol} \mathrm{dm}^{-3}$ and $298.15 \mathrm{~K}$. The correlation function are calculated under the integral equation closures $\mathrm{HNC}, \mathrm{HNC} / \mathrm{PY}$, and $\mathrm{HNC} / \lambda_{1}$ with the bridge parameter $B_{S}=0.047$, for which $u_{\mathrm{HNC} / \lambda_{1}}=u_{\mathrm{HNC}}$. (a): Like-sign (cation-cation or anion-anion) correlation function $g_{11}(r)$; (b): cationanion correlation function $g_{12}(r) . r$ is in units of $\AA$.

bridge parameter $B_{S}$ in the $\mathrm{HNC} / \lambda_{1}$ closure. An appealing alternative for establishing the value of $B_{S}$ that gives under $\mathrm{HNC} / \lambda_{1}$ improved results (relative to $\mathrm{HNC}$ and $\mathrm{HNC} / \mathrm{PY})$ is suggested by the correlation functions $g_{j l}(r)$ reported in figure 4 using $B_{S}=0.083$. For this value of the bridge parameter (at $c_{s}=0.02 \mathrm{~mol} \mathrm{dm}^{-3}$ ) $u_{\mathrm{HNC} / \lambda_{1}} \simeq u_{\mathrm{HNC}}=-1.280$. Inspection of figure 4 a reveals that at this value of $B_{S}$ there is only a minimal trace of the spurious peak in $g_{11}(r)$, with the overall shape of the function in better agreement with the simulation result $[5,6]$. At the same time figure $4 \mathrm{~b}$ shows that $g_{12}(r)$, although not as good as the result at $B_{S}=0.14$, is still an improvement over the predictions of HNC and HNC/PY $\left[\left(g_{12}^{\max }\right)_{\mathrm{HNC} / \lambda_{1}}=41.75\right.$ versus $\left(g_{12}^{\max }\right)_{\mathrm{HNC}}=37.6,\left(g_{12}^{\max }\right)_{\mathrm{HNC} / \mathrm{PY}}=33.7$, and $\left.\left(g_{12}^{\max }\right)_{\mathrm{MD}}=48.0\right]$. For the osmotic coefficient we find $\phi_{\mathrm{HNC} / \lambda_{1}}=\phi_{\mathrm{HNC} / \mathrm{PY}}=0.709$, which is in better agreement with $\phi_{\mathrm{MD}}=0.706$ than the $\mathrm{HNC}$ result $\phi_{\mathrm{HNC}}=0.713$.

We have tested this strategy for fixing the $B_{S}$ parameter of closure $\mathrm{HNC} / \lambda_{1}$ for solutions of the 2-2 electrolyte in the concentration range $0.001-0.5625 \mathrm{~mol}$ $\mathrm{dm}^{-3}$. The results obtained are summarized in table 1 . The second column in the table reports the value of $B_{S}$ for which $u_{\mathrm{HNC} / \lambda_{1}}=u_{\mathrm{HNC}}$ at each concentration. It is interesting that this "optimun" value of the bridge parameter does not change monotonically with the electrolyte concentration.

By following this procedure, the $\mathrm{HNC} / \lambda_{1}$ results for the reduced excess energy $u$ will be obviously equivalent to those of HNC; they are systematically smaller 
Table 1. Comparison of the predictions of the $\mathrm{HNC} / \lambda_{1}$ against the results by molecular dynamics, HNC, and HNC/PY calculations for model aqueous solutions of a 2-2 electrolyte at $298.15 \mathrm{~K}$. (a) $c_{s}$ is in units of $\mathrm{mol} \mathrm{dm}^{-3}$. (b) For each electrolyte concentration $B_{S}$ is selected such that $u_{\mathrm{HNC} / \lambda_{1}} \simeq u_{\mathrm{HNC}}$. (c) At $c_{s}=0.5625 \mathrm{~mol} \mathrm{dm}^{-3},\left(g_{12}^{\max }\right)_{\mathrm{MD}}$ and $\phi_{\mathrm{MD}}$ have not been reported; the data reported in the table are integral equation results from Duh and Haymet [6] based on bridge functions extracted from simulation data.

\begin{tabular}{|c|c|c|c|c|c|}
\hline $\mathbf{c}_{s}{ }^{a}$ & $\mathbf{B}_{S}{ }^{b}$ & $\mathbf{u}_{\mathrm{MD}}{ }^{c}$ & $\mathbf{u}_{\mathrm{HNC} / \lambda_{1}}$ & $\mathbf{u}_{\mathrm{HNC}}$ & $\mathbf{u}_{\mathrm{HNC} / \mathrm{PY}}$ \\
\hline 0.001 & 0.047 & -0.469 & -0.432 & -0.4328 & -0.4053 \\
\hline 0.005 & 0.096 & -0.9226 & -0.8283 & -0.8285 & -0.784 \\
\hline 0.020 & 0.083 & -1.413 & -1.280 & -1.280 & -1.245 \\
\hline 0.0625 & 0.040 & -1.834 & -1.713 & -1.713 & -1.697 \\
\hline 0.200 & 0.005 & -2.255 & -2.197 & -2.197 & -2.196 \\
\hline 0.5625 & 0.0 & -2.666 & -2.648 & -2.648 & -2.648 \\
\hline
\end{tabular}

\begin{tabular}{|c|c|c|c|c|c|}
\hline $\mathbf{c}_{s}^{a}$ & $\mathbf{B}_{S}{ }^{b}$ & $\phi_{\mathrm{MD}}{ }^{c}$ & $\phi_{\mathrm{HNC} / \lambda_{1}}$ & $\phi_{\mathrm{HNC}}$ & $\phi_{\mathrm{HNC} / \mathrm{PY}}$ \\
\hline 0.001 & 0.047 & 0.898 & 0.889 & 0.890 & 0.892 \\
\hline 0.005 & 0.096 & 0.808 & 0.800 & 0.802 & 0.802 \\
\hline 0.020 & 0.083 & 0.706 & 0.709 & 0.713 & 0.709 \\
\hline 0.0625 & 0.040 & 0.646 & 0.639 & 0.642 & 0.637 \\
\hline 0.200 & 0.005 & 0.611 & 0.593 & 0.594 & 0.592 \\
\hline 0.5625 & 0.0 & 0.638 & 0.615 & 0.615 & 0.615 \\
\hline
\end{tabular}

\begin{tabular}{|c|c|c|c|c|c|}
\hline $\mathbf{c}_{s}{ }^{a}$ & $\mathbf{B}_{S}{ }^{b}$ & $\left(\mathbf{g}_{12}^{\max }\right)_{\mathrm{MD}}{ }^{c}$ & $\left(\mathbf{g}_{12}^{\max }\right)_{\mathrm{HNC} / \lambda_{1}}$ & $\left(\mathbf{g}_{12}^{\max }\right)_{\mathrm{HNC}}$ & $\left(\mathbf{g}_{12}^{\max }\right)_{\mathrm{HNC} / \mathrm{PY}}$ \\
\hline 0.001 & 0.047 & 234 & 231 & 186 & 147 \\
\hline 0.005 & 0.096 & 112 & 104.7 & 85 & 69.6 \\
\hline 0.020 & 0.083 & 48 & 41.75 & 37.6 & 33.8 \\
\hline 0.0625 & 0.040 & 22.5 & 18.94 & 18.4 & 17.7 \\
\hline 0.200 & 0.005 & 9.6 & 8.82 & 8.81 & 8.77 \\
\hline 0.5625 & 0.0 & 5.02 & 4.73 & 4.73 & 4.73 \\
\hline
\end{tabular}


in magnitude than the corresponding molecular dynamics results. For the osmotic coefficient, HNC and HNC/PY are quite comparable, and the table shows that these two closures perform marginally better against simulation than the $\mathrm{HNC} / \lambda_{1}$ closure. On the other hand, the last part of the table reveals that $\mathrm{HNC} / \lambda_{1}$ performs considerably better than $\mathrm{HNC}$ and $\mathrm{HNC} / \mathrm{PY}$ with respect to the height $g_{12}^{\max }$ of the peak in the cation-anion radial distribution function. Especially encouraging are the results at the smallest concentrations $c_{s}=0.001$ and $0.005 \mathrm{~mol} \mathrm{dm}^{-3}$. To illustrate this point, in figure 5 we compare the $g_{j l}(r)$ at $c_{s}=0.001 \mathrm{~mol} \mathrm{dm}^{-3}$ calculated under closures $\mathrm{HNC}, \mathrm{HNC} / \mathrm{PY}$, and $\mathrm{HNC} / \lambda_{1}$ with $B_{S}=0.047$. From table 1 we see that $\left(g_{12}^{\max }\right)_{\mathrm{HNC} / \lambda_{1}}=231$ compares very well with $\left(g_{12}^{\max }\right)_{\mathrm{MD}}=234$. Inspection of figure $5 \mathrm{~b}$ reveals that the peak in $g_{12}(r)$ predicted by $\mathrm{HNC} / \lambda_{1}$ is slightly narrower than the peak calculated under HNC (and it is also narrower than the peak extracted from the molecular dynamics data [6]). With respect to the like-sign correlation function $g_{11}(r)$, the $\mathrm{HNC} / \lambda_{1}$ closure gives, compared against the simulation results, better results than $\mathrm{HNC} / \mathrm{PY}$. Unfortunately, for $B_{S}=0.047$, at which $u_{\mathrm{HNC} / \lambda_{1}}=u_{\mathrm{HNC}}$, $g_{11}(r)$ under closure $\mathrm{HNC} / \lambda_{1}$ displays the spurious peak closeby to $r \simeq 8 \AA$. The size of the peak, however, is much smaller than the peak in $g_{11}(r)$ under the HNC closure.

\section{Discussion}

Starting from the previously reported HNC/PY integral equation closure for solutions of binary electrolytes [7], we have proposed a very simple strategy for improving the closure performance concerning the structure and thermodynamic properties of the solution. Basically, the improvement is accomplished by adding new terms to the ion-ion bridge functions $b_{j l}(r)$ of the HNC/PY closure. The general idea is to borrow from the first few terms in the asymptotic expansion of the HNC/PY $c_{j l}(r)$ the specific functional form of the new terms in the bridge functions $b_{j l}(r)$. We note, however, that although the original HNC/PY work was concerned with general McMillan-Mayer level models of binary electrolytes, the present extension of the HNC/PY closure has been implemented in the present work only for restricted (or symmetric) models of electrolytes. In fact, by exploiting the symmetry of such electrolyte models, the discussion was actually cast in the language of the sum and difference functions introduced in equation (3).

The calculations presented in section 3.3 tested the predictions of the simplest extension, the $\mathrm{HNC} / \lambda_{1}$ closure, for aqueous solutions of a restricted 2-2 electrolyte. For this closure, only the sum combination $b_{S}(r)$ of ion-ion bridge functions is modified relative to the corresponding $\mathrm{HNC} / \mathrm{PY}$ bridge function; the difference bridge function $b_{D}(r)$ for this closure, like in the HNC and HNC/PY closures, is assumed to vanish at every $r$. The explicit of expression for $b_{S}(r)$ is given by equations $(21)$ and (27), and contains an unspecified parameter $B_{S}$. Initially we had hoped that the optimal value of $B_{S}$ would be fixed by demanding thermodynamic consistency for $\beta\left(\partial \pi / \partial \rho_{t}\right)_{T}$ when calculated through the compressibility and virial routes. While at every concentration studied we succeeded finding a $B_{S}$ for which the thermody- 
namic consistency is realized, the calculated correlation functions $g_{j l}(r)$ at those $B_{S}$ deviate substantially from the structure functions $g_{j l}(r)$ extracted from computer simulations. We were therefore forced to adopt the less elegant (but quite practical) criterion of choosing $B_{S}$ such that $u_{\mathrm{HNC} / \lambda_{1}}=u_{\mathrm{HNC}}$ is satisfied.

The performance of the $\mathrm{HNC} / \lambda_{1}$ closure based on this criterion for fixing $B_{S}$ was examined in section 3.3, where we concluded that, except for the osmotic coefficient, this new integral equation provides a substantial improvement over the HNC/PY and the HNC closures. Here we notice that, while still insisting on the approximation $b_{D}(r)=0$, there is room for improving the closure relation by further extending $b_{S}(r)$ with a 2-parameter $\lambda(r)$ function:

$$
\lambda(r)=\frac{B_{S}}{2} h_{D}(r) \chi(r)+\frac{B_{S}^{\prime}}{8} h_{D}(r)[\chi(r)]^{3} .
$$

The functional form of each term is borrowed from the respective term in the asymptotic expansion of $c_{S}(r)$ under the HNC/PY closure [the second term in equation (26)]. We refer to this closure by the acronym $\mathrm{HNC} / \lambda_{2}$, as its implementation requires the specification of the two bridge parameters $B_{S}$ and $B_{S}^{\prime}$.

When considered from the viewpoint of the ion-ion bridge functions $b_{j l}(r)$, the assumption $b_{D}(r)=0$ in the $\mathrm{HNC} / \lambda_{n}$ family of closures implies that under these closure relations $b_{11}(r)=b_{12}(r)$ applies. Ichiye and Haymet [13] and Duh and Haymet [6] have extracted the bridge functions for 2-2 electrolytes at several concentrations from computer simulations, and their results indicate that $b_{11}(r)$ and $b_{12}(r)$ have different sign, with $b_{12}(r)>0$. (See also Kjellander and Mitchell [14]). The bridge functions in the Ionic-Percus Yevick (IPY) integral equation closures developed by Ichiye and Haymet $[6,13,15]$ specifically account for this feature, and are able to account for the simulation results to a better degree than the $\mathrm{HNC} / \lambda_{1}$ closure described in section 3.3. It should be mentioned that the IPY closures have no free parameter. To our knowledge, the behaviour of these closures in the context of thermodynamic consistency between the various routes has not been examined. (The exponential $\exp \left[b_{12}(r)\right]$ in the IPY2 closure [15] contains a term with the factor $\exp \left[-\tau_{12}(r)\right]$, where $\tau_{12}(r)$ is an optimally designed $t_{12}(r)$ function. The presence of this $\exp \left[-\tau_{12}(r)\right]$ factor suggests that the range of the corresponding direct correlation function would become infinite as the electrolyte solution approaches the limit of material stability).

Only when we allow $b_{D}(r) \neq 0$ we can have $b_{11}(r)$ and $b_{12}(r)$ of different sign. This leads us to the $b_{D} / \lambda_{1}$ integral equation closure introduced in section 3.1. This closure requires the specification of the two bridge strength parameters $B_{S}$ and $B_{D}$. The criterions for fixing these parameters and the performance of this new integral equation are the subject of ongoing research in our group.

We conclude with the mention that, for the dilute systems studied here, the results of the $\mathrm{HNC} / \mathrm{PY}$ closure are practically identical to the results calculated with the Livshits-Martinov integral equation relation [16]. 


\section{Acknowledgements}

We dedicate this work to Jean-Pierre Badiali on the occasion of his 60th anniversary. F.O.R. and G.S. gratefully acknowledge the support of the Division of Chemical Sciences, Office of Basic Energy Sciences, Office of Energy Research, U.S. Department of Energy.

\section{References}

1. McMillan W.G., Mayer J.E. The statistical thermodynamics of multicomponent systems. // J. Chem. Phys., 1945, vol. 13, No. 7, p. 276-305.

2. Friedman H.L. A Course in Statistical Mechanics. Englewood Cliffs, New Jersey, Prentice-Hall, 1985.

3. Hansen J.-P., McDonald, I.R. Theory of Simple Liquids (2nd. edition). London, Academic Press, 1990.

4. Rossky P.J., Dudowicz J.B., Tembe B.L., Friedman H.L. Ionic association in model 2-2 electrolyte solutions. // J. Chem. Phys., 1980, vol. 73, No. 7, p. 3372-3383.

5. Smith D., Kalyuzhnyi Yu.V., Haymet A.D.J. Computer simulation of a model 2-2 electrolyte: multiple time-step molecular dynamics. // J. Chem. Phys., 1991, vol. 95, No. 12, p. 9165-9171.

6. Duh D.-M., Haymet A.D.J. Integral equation theory for charged liquids: model 2-2 electrolytes and the bridge function. // J. Chem. Phys., 1992, vol. 97, No. 10, p. 77167729 .

7. Raineri F.O., Friedman H.L., Stell G. Single-ion contributions to activity coefficient derivatives, second moment coefficients, and the liquid junction potential. // J. Solution Chem., 1999, vol. 28, No. 5, p. 463-488.

8. Xu H., Friedman H.L., Raineri F.O. Electrolyte solutions that unmix. Hydrophobic ions in water. // J. Solution Chem., 1991, vol 20, No. 8, p. 739-773.

9. Stell G. Criticality and phase transitions in ionic liquids. // J. Stat. Phys., 1995, vol 78, Nos. 1/2, p. 197-238.

10. Stillinger F., Lovett R. General restriction on the distribution of ions in electrolytes. // J. Chem. Phys., 1968, vol. 49, No. 5, p. 1991-1994.

11. Ng K.-C. Hypernetted chain solutions for the classical one-component plasma up to $\Gamma=7000$. // J. Chem. Phys., 1974, vol. 61, No. 7, p. 2680-2689.

12. Morris G.P., MacGowan D. Solution of the SSOZ equation for molecules of arbitrary symmetry. // Mol. Phys., 1986, vol. 58, No. 4, p. 745-761.

13. Ichiye T., Haymet A.J.D. Integral equation theory of ionic solutions. // J. Chem. Phys. 1990, vol. 93, No. 12, p. 8954-8962.

14. Kjellander R., Mitchell D.J. Dressed-ion theory for electrolyte solutions: a DebyeHückel-like reformulation of the exact theory for the primitive model. // J. Chem. Phys., 1994, vol. 101, No. 1, p. 603-626.

15. Babu C.S., Ichiye T. New integral equation theory for primitive model ionic liquids: from electrolytes to molten salts. // J. Chem. Phys., 1994, vol. 100, No. 12, p. $9147-$ 9155 .

16. Livshitzs A.I., Martinov G.A. The statistical theory of electrolyte melts. // J. Chem. Phys., 1989, vol. 90, No. 11, p. 6603-6609. 


\section{Новий клас місткових функцій для розчинів електролітів}

\section{Ф.О.Райнері, Дж.Стелл}

Державний університет Нью-Йорка, Стоні Брук, Нью-Йорк, США

Отримано 5 листопада 2001 р.

Для спрощених моделей розчинів електролітів ми представляємо новий набір замикань на рівні МакМіллана-Маєра, який покращує передбачення для парних кореляційних функцій іон-іон, отриманих у гіперланцюговому наближенні. Покращення здійснюється введенням простих функціональних форм для місткових функцій і деяких підгоночних параметрів, які відповідають різним критеріям. При нових умовах замикання, на противагу до гіперланцюгового наближення, пряма кореляційна функція "сума", що є важливою для визначення стійкості розв'язку по відношенню до фазового розділення, залишається скінченою при термодинамічних станах уздовж спінодалі і в критичній точці.

Ключові слова: розчини електролітів, спрощена модель, структура, термодинаміка, місткові функції, термодинамічна сумісність

PACS: 61.20.Gy, 61.20.Qg, 64.60.Fr, 64.70.Ja, 64.75. +g 